\title{
Optimization for a Flexure Hinge Using an Effective Hybrid Approach of Fuzzy Logic and Moth-Flame Optimization Algorithm
}

\author{
Minh Phung Dang, ${ }^{1}$ Hieu Giang Le, ${ }^{1}$ Ngoc Le Chau, ${ }^{2}$ and Thanh-Phong Dao $\mathbb{D}^{3,4}$ \\ ${ }^{1}$ Faculty of Mechanical Engineering, Ho Chi Minh City University of Technology and Education, Ho Chi Minh City, Vietnam \\ ${ }^{2}$ Faculty of Mechanical Engineering, Industrial University of Ho Chi Minh City, Ho Chi Minh City, Vietnam \\ ${ }^{3}$ Division of Computational Mechatronics, Institute for Computational Science, Ton Duc Thang University, \\ Ho Chi Minh City, Vietnam \\ ${ }^{4}$ Faculty of Electrical \& Electronics Engineering, Ton Duc Thang University, Ho Chi Minh City, Vietnam
}

Correspondence should be addressed to Thanh-Phong Dao; daothanhphong@tdtu.edu.vn

Received 17 December 2020; Revised 18 January 2021; Accepted 8 February 2021; Published 18 February 2021

Academic Editor: Zhen Song Chen

Copyright (c) 2021 Minh Phung Dang et al. This is an open access article distributed under the Creative Commons Attribution License, which permits unrestricted use, distribution, and reproduction in any medium, provided the original work is properly cited.

\begin{abstract}
Flexure hinge is a critical element in a positioner of a nanoindentation tester. To effectively work, a suitable flexure hinge should simultaneously meet multiple objectives, including rotation axis shift, safety factor, and angular deflection. The main aim of this article was to illustrate a hybrid method of the Taguchi method, fuzzy logic, response surface method, and Moth-flame optimization algorithm to solve the design optimization of a flexure hinge in order to enhance the three quality characteristics of the flexure hinge. Firstly, four common flexure hinges are compared together to seek the best suitable one. Secondly, numerical experiments are gathered via the Taguchi-based detasFlex software. Thirdly, three objective functions are transferred into signal to noises in order to eliminate the unit differences. Later on, fuzzy modeling is proposed to interpolate these three objective functions into one integrated objective function. An integrated regression equation is built using the response surface method. Finally, the flexure hinge is optimized by the Moth-flame optimization algorithm. The results found that the rotation axis shift is $10.944 * 10^{-5} \mathrm{~mm}$, the high safety factor is 2.993 , and the angular deflection is $52.0058 * 10^{-3} \mathrm{rad}$. The verifications are in a suitable agreement with the forecasted results. An analysis of variance and sensitivity analysis are also performed to identify the effects and meaningful contributions of input variables on the integrated objective function. In addition, employing the Wilcoxon signed rank test and the Friedman test, the results find that the proficiency of the proposed method has more benefits than the ASO algorithm and the GA. The results of this research provide a beneficial approach for conducting complicated multiobjective optimal problems.
\end{abstract}

\section{Introduction}

In general, machinery is created by a group of mechanical elements-assembled links. Mechanical elements are kinematic joints, e.g., gear, cam, and bearing [1]. There are many disadvantages of these joints such as friction, wear, and backlash. Therefore, it is difficult to achieve high accuracy. A current trend is that mechanical products are required for more compact size and better precision. For specifics, the commercial nanoindentation device is utilized for checking mechanical characteristics of material samples $[2,3]$, but its size is extremely large. As a result, they could not connect with the scanning electron microscope or transmission electron microscope to make in situ nanoindentation tester, which can monitor deformations and damages of materials online $[4,5]$. For this purpose, miniaturized devices are expected.

Currently, compliant mechanisms with flexure hinges are widely employed for accurate engineering systems [6-11]. Wadikhaye et al. proposed a compact serial- 
kinematic scanner applied to high-speed atomic force microscopy [12]. Gauthier and Piat developed a particular micro-macro positioning system for cell micromanipulation [13]. Zhu et al. proposed a XY nanopositioning stage integrated hydrid flexure amplifier with totally decoupled kinematics [14]. Lu et al. developed a flexure micro-gripper with high effectiveness and high precision for optical fiber assembly [15]. Huang et al. developed a compliant positioner for the nanoindentation tester [16]. Then, Huang et al. proposed a miniaturized modular nanoindentation device with the working travel of $12 \mu \mathrm{m}$ for the xy positioner and $40 \mu \mathrm{m}$ for the indenter [17]. Generally, in order to achieve the abovementioned working travels, the compliant positioners often integrate with compliant amplifiers [18-23]. Wang et al. proposed a hybrid amplifier of lever type and bridge type integrated into the micro-gripper for the grasping jaws to close and grasp the manipulated objects [24]. Tang et al. developed a novel hydraulic displacement amplifier for piezo-actuated large stroke precision positioning [25]. Chang and Du proposed a multiple Scott-Russell linkage to magnify the travel of PZT to achieve large output displacement [26]. Xu developed a new flexure gripper based on the Scott-Russell mechanism to amplify output displacement and to achieve a parallel motion of the gripper tips [27]. However, a limitation of the amplifiers is that the design space of device is enlarged. An alternative design way, the positioners should be integrated suitable flexure hinges. It is known that a nanoindentation tester often demands large working stroke, a small parasitic motion, a high safety factor, a good positioning accuracy, and a linear displacement. In order to simultaneously obtain the abovementioned characteristics, flexure hinges should possess a small rotation axis shift, a high safety factor, and a large angular deflection. The proposed flexure hinge can be integrated into a XY-stage for locating a thin material specimen, and into a Z-stage for driving an indenter.

Nowadays, flexure hinges are extremely effective elements for the precise stages because of no friction, no wear, and no backlash [28, 29]. In general, the quality of a compliant stage depends on the characteristics of the flexure hinge. In order to express the quality characteristics of flexure hinges, analytical approaches were proposed [30, 31]. In order to generate an initial shape of the flexure hinge, topology optimization was used [32]. In addition, to enhance the quality responses of flexure hinges, several other approaches such as shape and size optimization methods were proposed [33-35]. Although there have been much efforts with regard to designing and optimizing the flexure hinges, the design optimization of a flexure hinge for use in a nanoindentation tester has not been studied yet. Therefore, in the current research, a suitable flexure hinge is simultaneously optimized with multiple qualities, including rotation axis shift, safety factor, and angular deflection. The suggested flexure hinge can be embedded into both the $X Y$ and- $Z$ positioners in a nanoindentation tester.

It is known that three mentioned qualities of the flexure hinge are resisted together. In order to simultaneously balance the desires, the newly integrated optimization method is proposed to resolve the problem in this paper. In the last decades, the Taguchi method (TM) is widely utilized in quality enhancement with the lowest number of experiments [36]. Conversely, the TM only optimized a separate criterion, and it is not able to simultaneously solve multicriteria. Besides, there are many algorithms based on population such as genetic algorithm [37], cuckoo search algorithm [38], and particle swarm optimization [39]. However, these algorithms require initial control parameters. Therefore, in order to increase the convergence speed, other algorithms with less initial parameters were proposed such as the teaching-learning-based optimization [40-42], atom search optimization algorithm [43], Rao and Pawar's algorithms [44], and the Moth-flame optimization (MFO) [45]. These algorithms very effectively solved a fitness function. In order to effectively solve multiple fitness functions, a few integrated methods have been carried out by many researchers. For instance, Dao optimized a flexure mechanism through Taguchi, gray, and fuzzy logic [46]. Hou et al. proposed an integrated method of Taguchi, response surface method, and genetic algorithm to optimize one single criterion for the milling process [47]. Garg proposed hybrid algorithms for constrained optimal problems such as integration approach of PSO-GA [48] and GSA-GA $[49,50]$. Le proposed a game-theoretic optimization via Parallel MinMax Ant System algorithm, utilized to define the Nash equilibrium value in order to solve the confusion in selecting suitable bidders of multiround procurement trouble in software project management [51]. Dao et al. proposed an integrated method of Taguchi and cuckoo search algorithm for a flexure positioner [52]. Xiao et al. integrated the radius basis functional network and the genetic algorithm for a micro-gripper [53]. Dang et al. proposed an integrated approach of the Taguchi method, the response surface method, the enhanced adaptive neuro-fuzzy inference system, and the teaching-learning-based optimization for the positioning stage [54]. Tran et al. developed an integrated method for optimization of the Scott-Russell mechanism [55]. Furthermore, some different integration algorithms of Taguchi and fuzzy logic have been suggested [56]. In the mentioned optimization methods, the MFO algorithm is an efficient approach because of its fast convergence and decreased calculating time. However, this algorithm has not solved multiple performances yet. Hence, a combination of fuzzy logic and MFO algorithm is proposed for optimizing multiple fitness functions for the flexure hinge. In this research, an effective combination of the Taguchi method, fuzzy logic, the response surface method, and the MFO algorithm is developed to solve three objectives, namely, optimal problem of the flexure hinge in terms of accurate results, quick computing speed, and reducing costs.

The key aims of this article are to evaluate and choose a suitable flexure hinge for application in the nanoindentation tester. In this study, an integrated optimization method of the Taguchi method, fuzzy logic, the response surface method, and the MFO algorithm is developed to simultaneously optimize the three performances for the flexure hinge in terms of rotation axis shift, safety factor, and angular deflection. Numerically experimental datasets are collected based on the Taguchi and the detasFlex software. 
Three objective functions are then changed into signal to noises to eradicate their unit differences. Next, fuzzy modeling is to interpolate three objective functions into one integrated objective function. The integrated regression equation of the fuzzy modeling is formulated by the response surface method. Ultimately, the main geometrical parameters of the flexure hinge are optimized by the MFO algorithm. Sensitivity of input variables is evaluated the by analysis of variance to identify their influences. Lastly, the validations of the optimized results are validated.

\section{Proposed Optimization Methodology}

A flowchart illustrates a new effective optimization approach, which is integrated by the Taguchi method, fuzzy logic reasoning, the response surface method, and the MFO algorithm, as shown in Figure 1. In the field of flexure-based mechanisms, the parameters of flexure hinge are very sensitive to the three mentioned characteristics. The developed approach is proposed to optimize key geometrical parameters of the proposed flexure hinge in order to simultaneously achieve three technical requirements, including small rotation axis shift, high safety factor, and large angular deflection. In this study, the proposed approach comprises four main phases as follows: (i) define the optimal problem and select the flexure hinge, (ii) fuzzy modeling, (iii) establish the integrated regression equation by the response surface method, and (iv) conduct the optimization problem by the MFO algorithm.

\subsection{Phase (i): Define Optimal Problem and Select Flexure} Hinge. Based on the demands of a nanoindentation tester, the three abovementioned qualities of the flexure hinge are identified. Four common flexure hinges are evaluated by the destasFlex software [57]. Design variables and objective functions are determined. By a combination of the Taguchi method and the detasFlex Software, data are collected. Next, three objective functions are calculated to become corresponding signal to noises $(S / N)$ in order to eliminate the unit influences.

2.2. Phase (ii): Fuzzy Modeling. In this research, consequently, fuzzy modeling is proposed to interpolate three objective functions into one integrated objective function. The fuzzy modeling comprises knowledge base, fuzzification process, inference engine, and defuzzification procedure. Specifics could be concisely illustrated in the literature [58-60]. Firstly, the fuzzification process utilizes membership functions (MFs) to fuzzify the S/N proportions. Later on, the inference engine implements a reasoning replied the on fuzzy rules. Ultimately, defuzzification transforms a fuzzy value into an integrated objective function $(Z)$.

2.3. Main Step 1: Fuzzification Process. In the fuzzification process, real values are converted into linguistic parameters. In addition, knowledge-based rules are built, and membership functions (MF) of the fuzzy sets are determined.
Inference engine system relies on the established rules, which permits to utilize practical experiences from the designer in order to achieve high precision for the control system.

\subsection{Main Step 2: Defuzzification Process. Defuzzication} makes the output of the fuzzy inference system (FIS) into a real value, a nonfuzzy value $(Z)$, which is a combination of three fitness functions into one objective function. In this process, the centroid method is utilized for the conversion. Based on the integrated objective function, the regression equation was established to map the input design variables and the integrated objective function. To execute the FIS algorithm modeling, the outputs of the system are computed based on the centroid method, and Mamdani insinuation is utilized for defuzzification in this article. In this study, the Gaussian MFs are proposed for both the inputs and outputs in order to establish the fuzzy sets based on initial data. The value of MFs are in the range from zero to one. The Gaussian membership function is identified by:

$$
\mu_{A}(x, c, \sigma)=e^{-(1 / 2)((x-c) / \sigma)^{2}},
$$

where Gaussian MF is defined by $c$ and $\sigma$; $c$ symbolizes the MFs center; and $\sigma$ defines the MFs width.

2.5. Phase (iii): Establish Integrated Regression Equation. After the defuzzification process, based on the results of the three objective functions and design variables, the integrated regression equation was interpolated to establish the relation among the input variables and the integrated objective $(Z)$. If this equation is reliable, it is used for the optimization process in a later phase.

2.6. Phase (iv): Optimize Parameters by Moth-Flame Optimization. The MFO algorithm was proposed to solve many different engineering areas [45]. As illustrated in Figure 1, the MFO randomly initializes moths in the resolution area, then computing each moth's fitness values (i.e., position), as well as attaching the best position via flame. Later on, apprising the positions of moths relies on a helical travelling formula to obtain better points attached via a flame, upgrading the latest and the most perfect specific points, as well as replicating the prior procedures (i.e., improving the current positions of the moths and creating new ones) till the assessment objectives are satisfied. Features and applications of the MFO algorithm can be read in more literatures [61-63]. In addition, the initial setting parameters of this algorithm are illustrated in Table 1.

This MFO algorithm comprises three substeps as follows.

2.7. Substep 1: Producing the Initial Population of Moths. Every moth could wing in 1-D, 2-D, 3-D, or hyperdimensional area. An initial array of moths is defined as follows: 


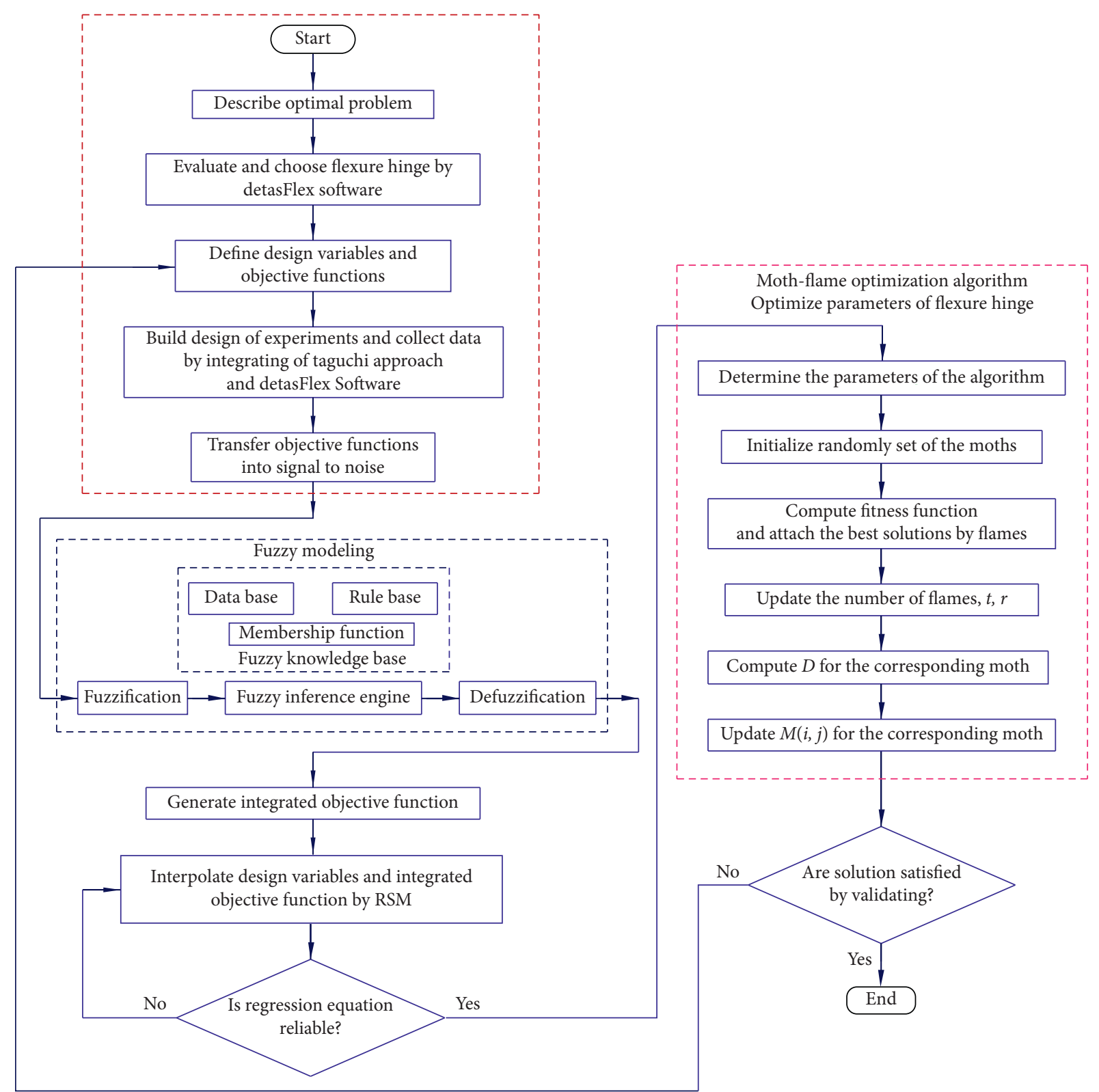

FIgURE 1: Flowchart for the proposed modeling and optimization method.

TABLE 1: Initial setting parameters of the MFO algorithm. 


$$
M=\left[\begin{array}{ccccc}
m_{1,1} & m_{1,2} & \cdots & \cdots & m_{1, d} \\
m_{2,1} & m_{2,2} & \cdots & \cdots & m_{2, d} \\
\cdot & \cdot & \cdot & \cdot & \cdot \\
\cdot & \cdot & \cdot & \cdot & \cdot \\
\cdot & \cdot & \cdot & \cdot & \cdot \\
m_{n, 1} & m_{n, 2} & \cdots & \cdots & m_{n, d}
\end{array}\right],
$$

where $n$ and $d$ are the moths' number as well as the number of dimensions in the resolution area, respectively. In addition, the suitability values for full moths are stored in an array as

$$
O M=\left[\begin{array}{c}
O M_{1} \\
O M_{2} \\
\cdot \\
\cdot \\
\cdot \\
O M_{n}
\end{array}\right]
$$

In the MFO algorithm, flames are regarded as the rest aspects. This matrix depicts the flames in the D-dimensional area monitored by their appropriateness formula vector:

$$
\begin{aligned}
F & =\left[\begin{array}{ccccc}
F_{1,1} & F_{1,1} & \cdots & \cdots & F_{1,1} \\
F_{1,1} & F_{1,1} & \cdots & \cdots & F_{1,1} \\
\cdot & \cdot & \cdot & \cdot & \cdot \\
\cdot & \cdot & \cdot & \cdot & \cdot \\
\cdot & \cdot & \cdot & \cdot & \cdot \\
F_{1,1} & F_{1,1} & \cdots & \cdots & F_{1,1}
\end{array}\right], \\
O F & =\left[\begin{array}{c}
O F_{1} \\
O F_{2} \\
\cdot \\
\cdot \\
\cdot \\
O F_{n}
\end{array}\right] .
\end{aligned}
$$

It is remarked that both solutions are generated by moths and flames. The way of handling and upgrading them in each iteration illustrates a dissimilarity among them. Until now, although the best position of the moths is achieved by the flames, the moths could be real investigation agents that transfer round the exploration space. Particularly, flames could be regarded as flags that are decreased via moths while investigating the exploration area. As a result, every moth investigates round a flame and upgrades the solution if obtaining a better position. The best solution of a moth can be never lost by this mechanism.

2.8. Substep 2: Upgrading the Moths' Positions. The MFO utilizes three different functions to congregate the global optimization of the optimal problems. These formulas are identified as

$$
\mathrm{MFO}=(I, P, T)
$$

where $I$ indicates the initial random positions of the moths. This function is expressed as follows:

$$
I: \varnothing \longrightarrow\{M, O M\} .
$$

The $P$ function illustrates the mobility of the moths in the search space. This function obtained the matrix of $M$ and returns with its upgraded one ultimately.

$$
P: M \longrightarrow M \text {. }
$$

The $T$ function represents completion of the investigation procedure. True if the assessment criterion is satisfied, and false if the assessment criterion is not satisfied.

$$
T: M \longrightarrow\{\text { true, false }\} \text {. }
$$

The formula of $I$ function is utilized for executing the random distribution.

$$
M(i, j)=(u b(i)-l b(j)) * r \text { and }+l b(j),
$$

where $l b$ and $u b$ illustrate the lower and upper bounds of design variables, respectively. As aforementioned, the moths fly in the investigation space utilizing the cross direction.

Firstly, helix's primary point ought to begin from the moth. Secondly, helix's ending point ought to be the position of the flame. Finally, fluctuation of the variety of spirals ought to not surpass the searching area. As a result, the logarithmic helix for the MFO algorithm is identified as:

$$
S\left(M_{i}, F_{j}\right)=D_{i} \cdot e^{b t} \cdot \cos (2 \pi t)+F_{j},
$$

where $D_{i}, b$, and $t$ express the gap of the $i$-th moth for the $j$-th flame, a stable value for determining the shape of the logarithmic spiral, and a random number from -1 to 1 , respectively. $D$ parameter is computed as follows:

$$
D_{i}=\left|F_{j}-M_{i}\right| \text {, }
$$

where $M_{i}, F_{j}$, and $D_{i}$ illustrate the $i$ th moth, the $j$-th flame, and the gap of the $i$ th moth for the $j$-th flame, respectively.

In the MFO, the equilibrizing among exploitation and exploration is ensured by the helical movement of the moth near the flame in the investigation area. In addition, to avoid dropping in the entraps of the local optimization, the optimized resolutions are preserved in each reiteration, and the moths hover around the flames so that every moth hovers nearby the nearest flame utilizing the OF and OM matrices.

2.9. Substep 3: Updating the Number of Flames. This part emphasizes improving the exploitation of the MFO algorithm (i.e., upgrading the moths' positions in $n$ different places in the investigation area can decline a chance of exploitation of the best hopeful resolutions). Consequently, reducing quantity of the flames assists to resolve this problem in accordance with the following equation: 


$$
\text { flame no }=\operatorname{round}\left(N-l * \frac{N-l}{T}\right),
$$

where $N, l$, and $T$ present the maximal number of flames, the current quantity of iterations, as well as the maximal quantity of iterations, respectively. These more details can be read in the literature [45].

In the present study, the hybrid approach combines the Taguchi method, fuzzy logic, the response surface method, and the MFO algorithm in order to optimize the three quality responses for the selected flexure hinge. In general, each objective function is assigned by the weight factor. Meanwhile, in this research, in the fuzzy modeling phase, three objective functions are interpolated into one integrated objective function and the weight factor for each objective function is also assigned automatically by fuzzy modeling. In addition, mechanical engineers can apply their experiences based on fuzzy modeling. As a result, this hybrid approach is effective, rapid, and accurate for solving the complex optimization problems.

There are several differences in the proposed method, compared to others. Firstly, an integrated optimization method of the Taguchi method, fuzzy logic, the response surface method, and the MFO algorithm is developed to simultaneously optimize the three performances for the flexure hinge. Especially, three objective functions are then changed into the $\mathrm{S} / \mathrm{N}$ values to eradicate their unit differences. Next, fuzzy modeling is to interpolate three objective functions into one integrated objective function. The integrated regression equation of the fuzzy modeling is established by the response surface method. Particularly, in this research, the weight factor is ignored by utilizing fuzzy modeling.

\section{Results and Discussion}

3.1. Evaluation and Selection for Flexure Hinge. The key target of this article is to seek a suitable flexure hinge for integrating into compliant positioners in the nanoindentation tester. To find suitable flexure hinge, three important assessment criteria, including rotation axis shift, safety factor, and maximal angular deflection, are interested. Figure 2 illustrates four common kinds of flexure hinges, including right circular hinge, corner-filleted hinge, elliptical hinge, and power function hinge (power of 2). To compare fairly the main characteristics, including rotation axis shift, safety factor, and angular deflection, the initial geometrical parameters $\left(H=10 \mathrm{~mm}, h=0.5 \mathrm{~mm}, \quad L_{1}=10 \mathrm{~mm}\right.$, $L_{2}=10 \mathrm{~mm}$, and $w=5 \mathrm{~mm}$ ) were selected with the same general dimensions. In addition, the four proposed flexure hinges were established with the same left fixed constraint and free end with the same initial input condition deflection angle $\left(\varphi=1^{0}\right)$. For specifics, the rotational axis shift, $v$, is the distance between the primary center point $C$ and the directed center point $C$, with an unchanging distance of $L_{2}$ during the movement (fixed center approach), as illustrated in Figure 3. The axis shift defines the rotational precision. The rotational axis shift of a flexure hinge is desired to achieve the smallest value in order to obtain a high positioning accuracy. Furthermore, a safety factor is defined in accordance with the relation between yield strength and maximal stress. The final characteristic, the maximum angular deflection can be expressed by

$$
\varphi_{\max }=S_{F} \cdot \varphi
$$

where $S_{F}$ is the safety factor and $\varphi$ is the input deflection angle.

Moreover, safety factor and maximal angular deflection are two important output responses for ensuring the strength of the material and expanding the working stroke of the positioning stage due to the limitation of a piezoactuator. As shown in Table 2, the results indicated that the elliptical hinge has the rotation axis shift of $9.7633 e-05 \mathrm{~mm}$, safety factor of 2.95, and a maximal angular deflection of $0.052 \mathrm{rad}$. The rotation axis shift of the elliptical hinge is higher than that of the power function 1 (power of $n=2$ ) and the right circular hinge but smaller than that of a corner-filleted hinge. In addition, safety factor and maximal angular detection of the elliptical hinge achieved the highest values. Therefore, the elliptical hinge was selected as the best suitable hinge, which is expected to integrate into positioners in the nanoindentation tester.

Meanwhile, rotation axis shift, safety factor, and maximal angular deflection are conflicted together. Consequently, to achieve simultaneously the output quality responses, the hybrid optimization approach was proposed to balance for the three output responses in this article.

\subsection{Determination of the Design Optimization for the Flexure} Hinge. According to the field of flexure-based mechanisms [6-15], the main geometrical parameters of the flexure hinge significantly affect the output quality of the responses. In this paper, three geometrical parameters of the elliptical flexure hinge include the thickness of $(h), x$-axis radius $\left(r_{x}\right)$, and $y$ axis radius $\left(r_{y}\right)$, which were chosen as the design variables. Consequently, with the purpose of improving the output objectives of the flexure hinge, these key geometric factors of the flexure hinge need to be optimized. In order to be used for positioners, the elliptical hinge should meet important characteristics such as

(a) A small rotation axis shift for increasing the accuracy in linear motion

(b) A high safety factor for ensuring safety operation

(c) A large angular deflection for enlarging spacious ability of location

3.2.1. Design Variables. Three key geometric dimensions of the elliptical hinge consist of the thickness $(h)$, the $x$-axis radius $\left(r_{x}\right)$, and the $y$-axis radius $\left(r_{y}\right)$, as shown in Figure 3 . The vector of design variables is expressed as $X=\left[h, r_{x}, r_{y}\right]^{T}$. The limitation requirements for the design variables were formed according to specialized knowledge, machining ability, and the mechanical engineer's experiences, which were stated by 


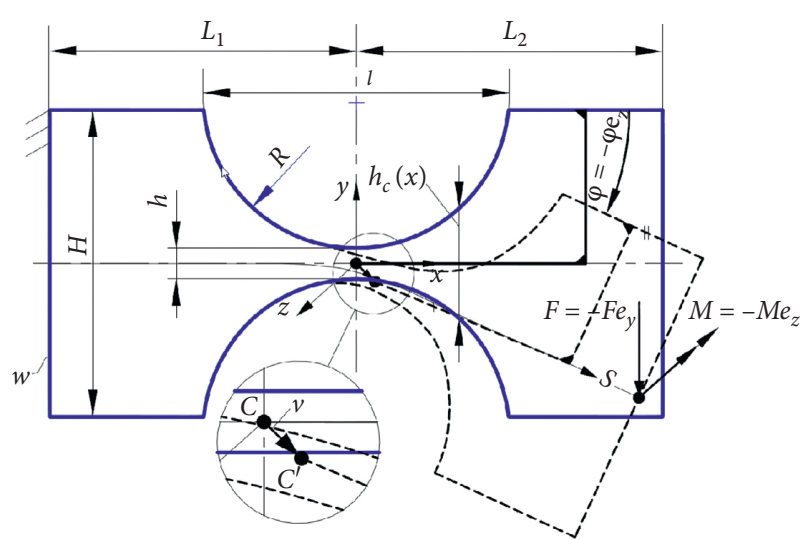

(a)

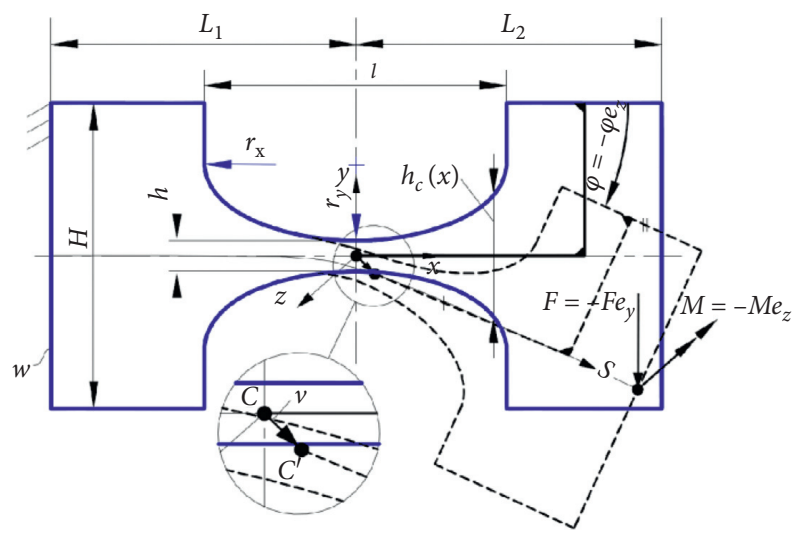

(c)

FIGURE 2: Four types of flexure hinges [57]. (a) Right circular hinge.

$$
\left\{\begin{array}{l}
0.4 \mathrm{~mm} \leq h \leq 0.6 \mathrm{~mm}, \\
4 \mathrm{~mm} \leq r_{x} \leq 6 \mathrm{~mm}, \\
2 \mathrm{~mm} \leq r_{y} \leq 3 \mathrm{~mm},
\end{array}\right.
$$

where $h, r_{x}$, and $r_{y}$ are the thickness of the elliptical flexure hinge, the $x$-axis radius, and the $y$-axis radius, respectively.

3.2.2. Objective Functions. In this paper, the three quality objectives of the elliptical hinge are regarded as: (i) The small rotation axis shift, $y_{1}(X)$, is required to ensure the position precision. (ii) The high safety factor, $y_{2}(X)$, is expected to guarantee the strength of the flexure hinge. (iii) The angular deflection, $y_{3}(X)$, is desired to be as large as possible so as to enlarge the operating stroke ability. To conclude, the optimization problem is illustrated as follows:

Find $X=\left[h, r_{x}, r_{y}\right]^{T}$

$$
\begin{aligned}
& \operatorname{Min} y_{1}(X), \\
& \operatorname{Max} y_{2}(X), \\
& \operatorname{Max} y_{3}(X)
\end{aligned}
$$

It is difficult to solve this multi-criteria problem for three objective functions. In this research, these objective functions

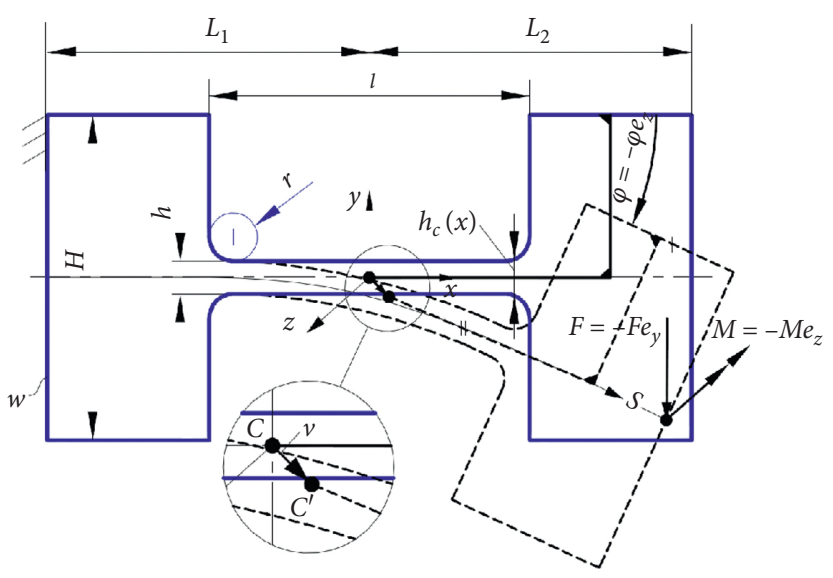

(b)

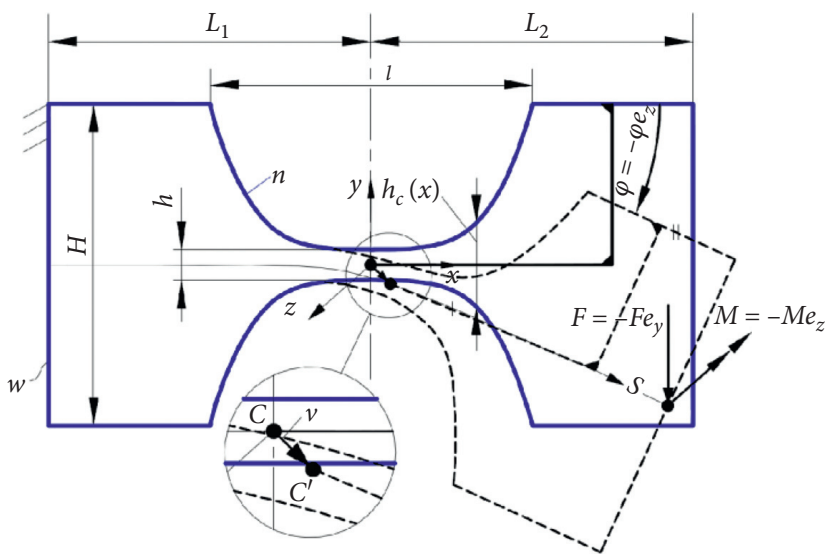

(d)

(b) Cornear-filleted hinge. (c) Elliptical hinge. (d) Power function hinge.

were transferred into signal to noise in order to eliminate the unit differences of the three objective functions. Later on, based on the signal to noise results of the three objective functions, the fuzzy modeling was utilized to integrate three objective functions into one objective function. And then, the multi-criteria problem was transformed into one criterion optimization problem. In the Taguchi analysis, the more signal to noise it obtains, the better output objective function it achieves. On the other hand, to find optimal parameters, the integrated function is expected to achieve maximal value in order to achieve a high $S / N$ ratio. Therefore, in this study, the optimal problem for an integrated objective function was defined as

$$
\operatorname{Max} Z(X) \text {. }
$$

3.2.3. Constraints. The elliptical hinge is expected to integrate into positioners for a nanoindentation tester. It was working under an elastic limitation of the suggested material, which is expressed by

$$
g(x)=\sigma \leq \frac{\sigma_{y}}{S_{F}},
$$

where $\sigma_{y}$ is the yield stress of the material, namely, AL7075 and $S_{F}$ is the safety factor. 


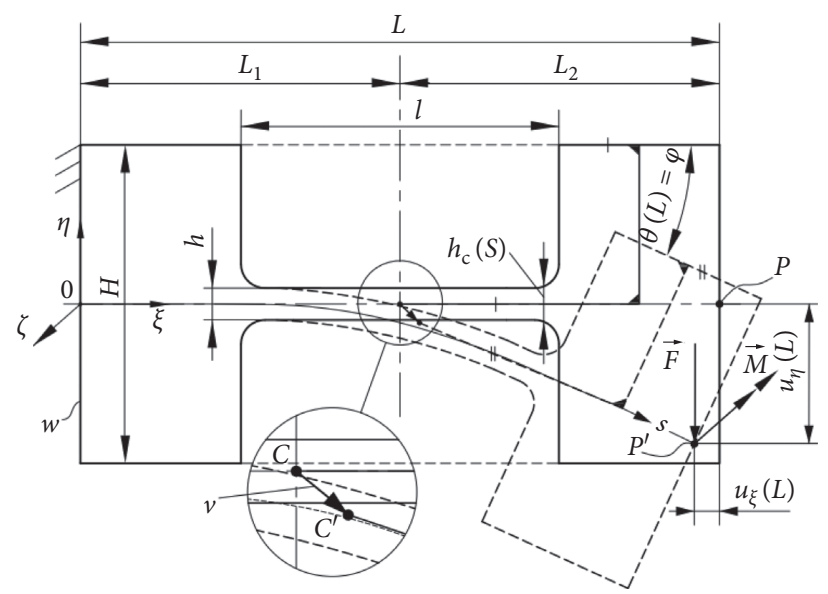

Figure 3: Factors for the theoretical characterization of a flexure joint (illustration of the primary and deflected position) for the identification of the rotational axis shift [57].

TABle 2: Specifications of the four flexure hinges.

\begin{tabular}{|c|c|c|c|c|c|}
\hline Flexure hinges & Geometrical parameters $(\mathrm{mm})$ & $\begin{array}{l}\text { Input deflection } \\
\text { angle (degree) }\end{array}$ & $\begin{array}{l}\text { Rotation axis shift } \\
\quad(v)(\mathrm{mm})\end{array}$ & $\begin{array}{c}\text { Safety } \\
\text { factor }(S F)\end{array}$ & $\begin{array}{l}\text { Maximal angular deflection } \\
(\varphi)(\mathrm{rad})\end{array}$ \\
\hline $\begin{array}{l}\text { Right circular } \\
\text { hinge }\end{array}$ & $\begin{aligned} H=10, h & =0.5, L_{1}=10, L_{2}=10, \\
l & =9.987, w=5\end{aligned}$ & Analysis angle $\left(1^{\circ}\right)$ & $7.2113 e-05$ & 2.11 & 0.037 \\
\hline $\begin{array}{l}\text { Corner filleted } \\
\text { hinge }\end{array}$ & $\begin{array}{c}H=10, h=0.5, L_{1}=10, L_{2}=10, \\
l=10, w=5\end{array}$ & Analysis angle $\left(1^{\circ}\right)$ & 0.00022379 & 0.1 & 0.176 \\
\hline Elliptical hinge & $\begin{array}{c}H=10, h=0.5, L_{1}=10, L_{2}=10 \\
\quad l=10, w=5, r_{x}=5, r_{y}=2.5\end{array}$ & Analysis angle $\left(1^{\circ}\right)$ & $9.7633 e-05$ & 2.95 & 0.052 \\
\hline $\begin{array}{l}\text { Power function } \\
1(n=2)\end{array}$ & $\begin{array}{c}H=10, h=0.5, L_{1}=10, L_{2}=10, \\
l=10, w=5\end{array}$ & Analysis angle $\left(1^{\circ}\right)$ & $5.486 e-05$ & 1.55 & 0.027 \\
\hline
\end{tabular}

The rotation axis shift was desired to be smaller than 0.00012 as

$$
y_{1}(X) \leq 0.00012
$$

The safety factor was required to be higher than 2 as

$$
y_{2}(X) \geq 2 \text {. }
$$

The angular deflection was preferred to be more than $0.052 \mathrm{rad}$ as

$$
y_{3}(X) \geq 0.05
$$

where $y_{1}, y_{2}$, and $y_{3}$ represent rotation axis shift, safety factor, and angular deflection, respectively.

\subsection{Establishment for Design of Experiments and Calculation} of $S / N$ Ratios. In this study, three geometrical parameters, including the thickness of the elliptical flexure hinge $(h)$, the $x$-axis radius $\left(r_{x}\right)$, and the $y$-axis radius $\left(r_{y}\right)$, were chosen as design variables. A design of experiments (DoE) was employed to determine the relation among input design variables and the quality responses. Each of the three factors was divided into three levels relying on the professional experiences of mechanical researchers, as demonstrated in Table 3. The $\mathrm{L}_{9}\left(3^{4}\right)$ experimental matrix of the TM was adopted to build a minimal experiment. Rotation axis shift $\left(y_{1}\right)$, safety factor $\left(y_{2}\right)$, and maximal angular deflection $\left(y_{3}\right)$
TABle 3: Process factors and their levels (unit: $\mathrm{mm}$ ).

\begin{tabular}{lcccc}
\hline Factors & Range & Level 1 & Level 2 & Level 3 \\
\hline$h$ & $0.4-0.6$ & 0.4 & 0.5 & 0.6 \\
$r_{x}$ & $4-6$ & 4 & 5 & 6 \\
$r_{y}$ & $2-3$ & 2 & 2.5 & 3 \\
\hline
\end{tabular}

were collected based on the detasFlex software, and results of the numerical experiment are indicated in Table 4.

Next, the three objective functions of the elliptical hinge were changed into the corresponding $S / N$ ratios as follows.

The $S / N$ for the larger type was utilized for the high safety factor and the large angular deflection, which was determined by

$$
\eta=-10 \log \left(\frac{1}{n} \sum_{i=1}^{n} \frac{1}{y_{i}^{2}}\right)
$$

The $S / N$ for the smaller type was utilized for the rotation axis shift, which was identified by

$$
\eta=-10 \log \left(\frac{1}{n} \sum_{i=1}^{n} y_{i}^{2}\right),
$$

where $y_{i}$ is the $i^{\text {th }}$ response and $n$ is the replication of $i^{\text {th }}$ experiment.

Based on the numeric experimental results in Table 4, the $S / N$ ratio values for $y_{1}, y_{2}$, and $y_{3}$ were calculated according 
TABLE 4: Experimental results and output responses.

\begin{tabular}{lcccccc}
\hline No. & $h(\mathrm{~mm})$ & $r x(\mathrm{~mm})$ & $r y(\mathrm{~mm})$ & $y_{1}(\mathrm{~mm})$ & $y_{2}$ & $y_{3}(\mathrm{rad})$ \\
\hline 1 & 0.4 & 4 & 2 & $7.8076 E-05$ & 0.052 \\
2 & 0.4 & 5 & 2.5 & $8.85 E-05$ & 3.319 & 0.058 \\
3 & 0.4 & 6 & 3 & $9.79 E-05$ & 3.65 & 0.064 \\
4 & 0.5 & 5 & 3.5 & $7.84 E-05$ & 2.36 & 0.041 \\
5 & 0.5 & 6 & 2 & $9.03 E-05$ & 0.047 \\
6 & 0.5 & 4 & 3 & 7.00012828 & 3.94 \\
7 & 0.6 & 5 & 2 & 0.00011544 & 0.069 \\
8 & 0.6 & 6 & 2.5 & 0.00012636 & 2.97 & 0.034 \\
9 & 0.6 & & & & 3.22 \\
\hline
\end{tabular}

to equations (21) and (22). Therefore, the orthogonal array with design variables, experimental results, and the $S / N$ ratio values, respectively, are shown in Table 5.

3.4. Establishment of the Fuzzy Model. The objective of the suggested fuzzy process is to transfer three inputs into one input in which the inputs are $S / N$ values of three performances of the elliptical hinge, and the output is considered as the integrated fitness function $(Z)$. The $Z$ function is then utilized for optimizing.

Based on the datasets of the design variables and the output responses, the Gaussian MFs were proposed for the fuzzy inputs and the output. The membership functions of the $S / N$ of the rotation axis shift, the $S / N$ of the safety factor, and the $S / N$ of the angular deflection are plotted in Figures 4(a)-4(c), respectively. Moreover, in accordance with the design experience, the input variables and the output responses are fuzzified into twenty-seven fuzzy sets: small small small (SM), small small medium (M), small small large (ML), small medium small (M), small medium medium (ML), small medium large (L), small large large (VL), medium small small (SM), medium small medium (SM), medium small large $(\mathrm{M})$, medium medium small (SM), medium medium medium (M), medium medium large $(\mathrm{ML})$, medium large small $(\mathrm{M})$, medium large medium (ML), medium large large, large small small (VS), large small medium (SM), large small large (SM), large medium small (S), large medium medium (SM), large medium large (M), large large small (SM), large large medium (M), and large large (ML). The related MFs for the input variables and the output variables are illustrated in Figure 5, and the particularized parameters of the fuzzy inference system with the Gaussian MFs are specified in Table 6. The output values of fuzzy modeling are given in Table 7 . Figures 6-8 illustrate correlations of $S / N$ inputs versus the output of this system. Figure 9 is an illustrative example of fuzzy rules.

3.5. Establishment of the Regression Equation. After establishing the integrated function based on fuzzy modeling, in order to find optimal parameters of the flexure hinges, an integrated regression equation was formed to map the input parameters and the integrated function. Regression equation illustrates the relationship design variables, and the output fuzzy is as follows:
TABLE 5: Results of the $S / N$ ratio for $y_{1}$ (SNRA1), $y_{2}$ (SNRA2), and $y_{3}$ (SNRA3).

\begin{tabular}{lcccccc}
\hline No. & $h$ & $r_{x}$ & $r_{y}$ & SNRA1 $(\mathrm{dB})$ & SNRA2 $(\mathrm{dB})$ & SNRA3 $(\mathrm{dB})$ \\
\hline 1 & 0.4 & 4 & 2 & 82.1496 & 9.3964 & -25.6799 \\
2 & 0.4 & 5 & 2.5 & 81.0605 & 10.4201 & -24.7314 \\
3 & 0.4 & 6 & 3 & 80.1868 & 11.2459 & -23.8764 \\
4 & 0.5 & 4 & 2.5 & 82.1139 & 7.4582 & -27.7443 \\
5 & 0.5 & 5 & 3 & 80.8830 & 8.6594 & -26.5580 \\
6 & 0.5 & 6 & 2 & 77.8368 & 11.9099 & -23.2230 \\
7 & 0.6 & 4 & 3 & 82.0607 & 5.8893 & -29.3704 \\
8 & 0.6 & 5 & 2 & 78.7529 & 9.4843 & -25.6799 \\
9 & 0.6 & 6 & 2.5 & 77.9678 & 10.1571 & -25.0362 \\
\hline
\end{tabular}

$$
\begin{aligned}
Z= & -2.058+3.518 \times h+0.5670 \times r_{x}+0.05367 \times r y-1.023 \times h \times h \\
& -0.01867 r_{x}^{2}+0.02067 \times r_{y}^{2}-0.2520 \times h \times r_{x}-0.7227 \times h \times r_{y} .
\end{aligned}
$$

Table 8 illustrates the ANOVA results of the integrated regression equation. This analysis was accomplished at 5\% significance and 95\% confidence levels. As illustrated in Table 8 , the percentage of the integrated regression equation of $r_{x}$ was the highest with $85.25 \%$, and its percentage of $r_{y}$ and $h$ were meaningfully greater at $9.22 \%$ and $3.76 \%$, respectively. However, the other influence parameters itemized gave a much smaller effect percentage. The percentage of collaboration between $r_{x}$ and $r_{x}$, between $r_{y}$ and $r_{y}$, and between $h$ and $h$ were $0.52 \%, 0.12 \%$, and $0.04 \%$, respectively. Consequently, to increase the value of $Z$, parameters $r_{x}$ and $r_{y}$ need to be extremely controlled. Furthermore, the percentage of error was $0 \%$ for $Z$.

The statistic method exploited the sensitivity of the inputs to the output-integrated response. As shown in Figure 10, factor $h$ demonstrated that in the range from $0.4 \mathrm{~mm}$ to $0.6 \mathrm{~mm}$, this factor influenced the output-integrated response to decrease marginally. Similarly, factor $r_{y}$ demonstrated that in the range from $2 \mathrm{~mm}$ to $3 \mathrm{~mm}$, it caused gradual reduction to the response. Meanwhile, the parameter $r_{x}$ depicted that in the range from $4 \mathrm{~mm}$ to $6 \mathrm{~mm}$, it affected a sharp increase to the output-integrated response.

Briefly, the overall influences of the input variables were demonstrated, as in Figure 11. It provides a rise as well as reduces the variety in each parameter. Based on this effect, the mechanical researchers can adjust the parameters in order to gain a suitable design for the proposed flexure hinge. 


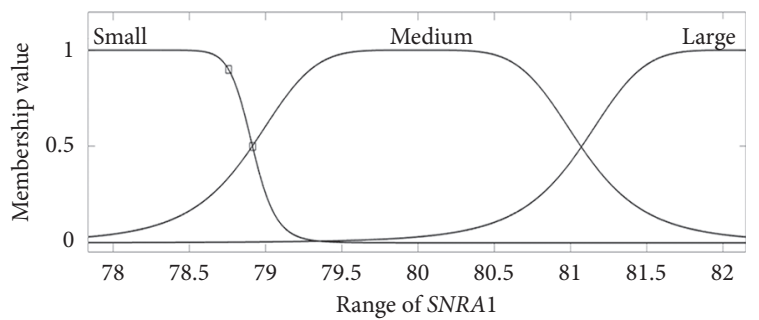

(a)

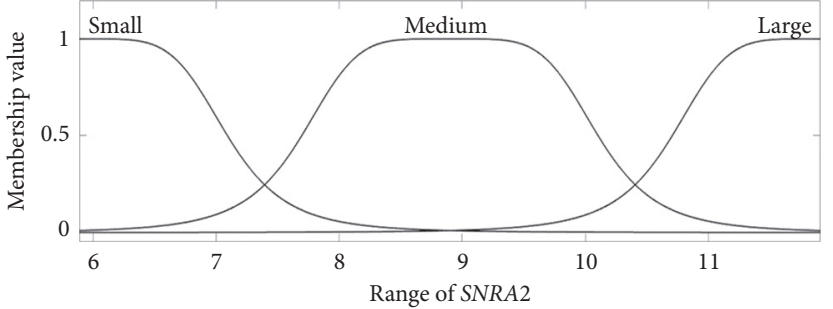

(b)

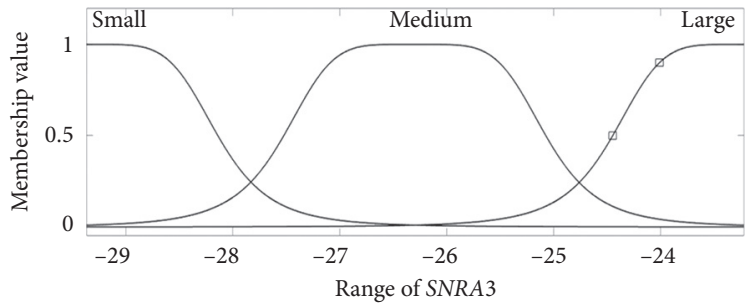

(c)

FIGURE 4: Membership functions plot: (a) rotation axis shift, (b) safety factor, and (c) angular deflection.

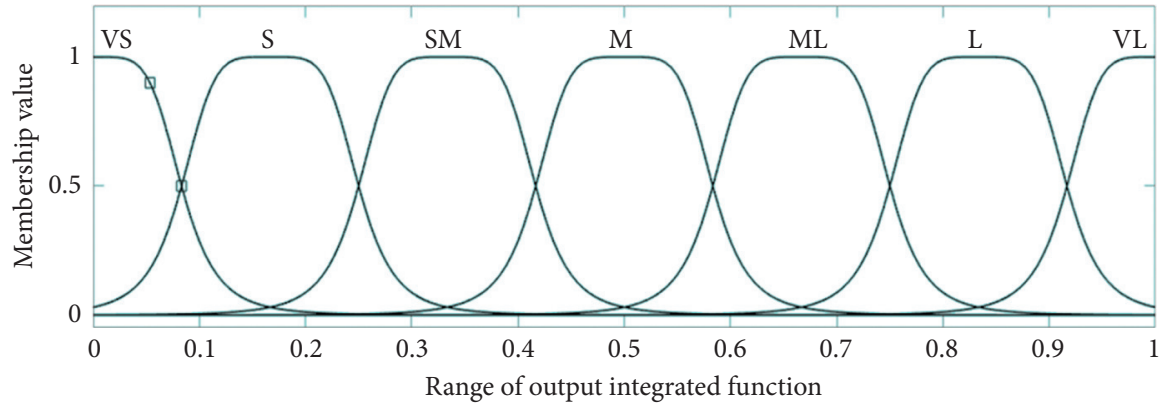

FIGURE 5: Membership functions plot for the output-integrated function.

3.6. Optimization Implementation. Based on the regression equation, the MFO algorithm was exploited for seeking optimization variables. The MFO algorithm was extended to seek the best parameters of the elliptical hinge because of its fast convergence speed to global optimization solutions.

In order to maximize the integrated function $(Z)$, the MFO algorithm was conducted by MATLAB 2017b. The primary parameters of MFO are illustrated in Table 9.

The optimal design variables were detected at $x_{\mathrm{val}}=[0.66$ $3]$ and $f_{\text {val }}=2.7614$. The optimal results were at $h=0.6 \mathrm{~mm}$, $r_{x}=6 \mathrm{~mm}, r_{y}=3 \mathrm{~mm}$, and $Z=2.7614$ corresponding to $y_{1}=0.00010944 \mathrm{~mm}, y_{2}=2.99349$, and $y_{3}=0.0520058 \mathrm{rad}$, respectively.

3.7. Validation. In the previous section, the optimal factors are found, including $\mathrm{h}=0.6 \mathrm{~mm}, \mathrm{rx}=6 \mathrm{~mm}$, ry $=3 \mathrm{~mm}$. With $\mathrm{Z}=2.7614$, the optimal performances of the hinge are corresponding to $\mathrm{y} 1=0.00010944 \mathrm{~mm}, \mathrm{y} 2=2.99349$, and y3 $=0.0520058 \mathrm{rad}$. By using the optimal factors, the optimal performanaces of the hinge are confirmed through detasFlex software. Later on, numerical validations were carried out to verify the forecasted results. This procedure was implemented with the same constraints and the same input initial angle. The verified results illustrated that the rotation axis shift was approximately $0.00011715 \mathrm{~mm}$, the safety factor was 2.95, and the angular deflection was $0.052 \mathrm{rad}$.

Table 10 depicts that the error among forecasted consequences and confirmations for the rotation axis shift, the safety factor, and the angular deflection is $6.58 \%, 1.47 \%$ and $0.01 \%$, respectively. It means that the predicted solutions are in a suitable agreement with the confirmed consequences.

As illustrated in Table 11, the optimization results were better than the initial results about the safety factor and the angular deflection in comparison with the initial design. Specifically, an enhancement for the safety factor was approximately $10.46 \%$ and for angular deflection it was about $10.65 \%$. However, a reduction for the rotation axis shift was approximately $21.2 \%$. It depicts that the proposed integrated approach is an effective method to carry out multi-criteria optimal problem of the proposed flexure hinge.

In addition, as illustrated in Table 12, the optimization results of the flexure hinge were compared again with the results of the right circular hinge, the corner-filleted hinge, the and power function $1(n=2)$. The results showed that the optimal hinge obtained the highest value of the safety factor and the maximal angular deflection. As a result, the optimal hinge will be chosen for integration into compliant 
TABLE 6: Fuzzy rules for evaluating the characteristics of the elliptical flexure hinge.

\begin{tabular}{|c|c|c|c|c|}
\hline Rule & $\begin{array}{c}\text { Input } 1 \\
\text { Rotation axis shift }\end{array}$ & $\begin{array}{c}\text { Input } 2 \\
\text { Safety factor }\end{array}$ & $\begin{array}{c}\text { Input } 3 \\
\text { Angular deflection }\end{array}$ & Output \\
\hline $\mathrm{R} 1$ & Small & Small & Small & SM \\
\hline $\mathrm{R} 2$ & Small & Small & Medium & $\mathrm{M}$ \\
\hline $\mathrm{R} 3$ & Small & Small & Large & ML \\
\hline $\mathrm{R} 4$ & Small & Medium & Small & $\mathrm{M}$ \\
\hline R5 & Small & Medium & Medium & ML \\
\hline R6 & Small & Medium & Large & $\mathrm{L}$ \\
\hline R7 & Small & Large & Small & ML \\
\hline $\mathrm{R} 8$ & Small & Large & Medium & $\mathrm{L}$ \\
\hline R9 & Small & Large & Large & $\mathrm{VL}$ \\
\hline $\mathrm{R} 10$ & Medium & Small & Small & SM \\
\hline R11 & Medium & Small & Medium & SM \\
\hline $\mathrm{R} 12$ & Medium & Small & Large & $\mathrm{M}$ \\
\hline $\mathrm{R} 13$ & Medium & Medium & Small & SM \\
\hline R14 & Medium & Medium & Medium & $\mathrm{M}$ \\
\hline R15 & Medium & Medium & Large & ML \\
\hline R16 & Medium & Large & Small & $\mathrm{M}$ \\
\hline R17 & Medium & Large & Medium & ML \\
\hline R18 & Medium & Large & Large & $\mathrm{L}$ \\
\hline R19 & Large & Small & Small & VS \\
\hline R20 & Large & Small & Medium & SM \\
\hline $\mathrm{R} 21$ & Large & Small & Large & SM \\
\hline R22 & Large & Medium & Small & S \\
\hline $\mathrm{R} 23$ & Large & Medium & Medium & SM \\
\hline $\mathrm{R} 24$ & Large & Medium & Large & $\mathrm{M}$ \\
\hline $\mathrm{R} 25$ & Large & Large & Small & SM \\
\hline R26 & Large & Large & Medium & $\mathrm{M}$ \\
\hline $\mathrm{R} 27$ & Large & Large & Large & ML \\
\hline
\end{tabular}

TABLE 7: The inputs and the output of fuzzy modeling.

\begin{tabular}{lccc}
\hline SNRA1 & SNRA2 & SNRA3 & Output $(Z)$ \\
\hline 82.1496 & 9.3964 & -25.6799 & 0.363 \\
81.0605 & 10.4201 & -24.7314 & 0.59 \\
80.1868 & 11.2459 & -23.8764 & 0.79 \\
82.1139 & 7.4582 & -27.7443 & 0.27 \\
80.8830 & 8.6594 & -26.5580 & 0.446 \\
77.8368 & 11.9099 & -23.2230 & 0.886 \\
82.0607 & 5.8893 & -29.3704 & 0.0946 \\
78.7529 & 9.4843 & -25.6799 & 0.619 \\
77.9678 & 10.1571 & -25.0362 & 0.686 \\
\hline
\end{tabular}

positioners applied to nanoindentation tester system based on the quality responses of the proposed hinge.

3.8. Comparison with Different Methods. In this research, to assess the statistical manner of the integrated method, a statistic analysis was utilized. The Wilcoxon test was utilized to illustrate the manner of the integrated method. The calculation simulations were accomplished 30 times for every method in seeking the integrated objective function $(Z)$. This technique was accomplished at 5\% meaningful grade and $95 \%$ assurance intervals. The outcomes of the Wilcoxon test are indicated in Table 13.

As known, a null hypothesis is supposed when there is no major dissimilarity between the average values of the two algorithms. The results illustrate that the $P$ value is smaller than 0.001 ( $5 \%$ significance level), which is a great confirmation against the null hypothesis, as illustrated in Table 13. It means that there is statistic dissimilarity among the hybrid approach and the atom search optimization (ASO) algorithm [43]. The result indicates that the integrated approach overcomes the ASO algorithm in resolving the optimal design of the proposed flexure hinge.

In a different way, the Friedman test determined the dissimilarity between the proposed integrated optimization method and the ASO algorithm at a meaningful grade of $\alpha=0.05$. The Friedman test was implemented for the integrated objective function. The computational simulations were carried out 30 times for every algorithm. The consequences illustrated that the $P$ value is smaller than 0.001 . Consequently, the null hypothesis was denied. Therefore, there is dissimilarity among the proposed integrated method and the ASO algorithm, as shown in Table 14.

In addition, the average convergence time for 30 runs of each algorithm was compared among the proposed method and the genetic algorithm (GA) [37] and the ASO algorithm [43] for seeking the integrated objective function. All three methods were run with a maximum number of iterations of 500. The result showed that the convergence speed of the proposed method overcomes the convergence speed of the ASO algorithm and the GA algorithm, as illustrated in Table 15.

In summary, the major findings of the present article are as follows: (i) An elliptical hinge is the most suitable 


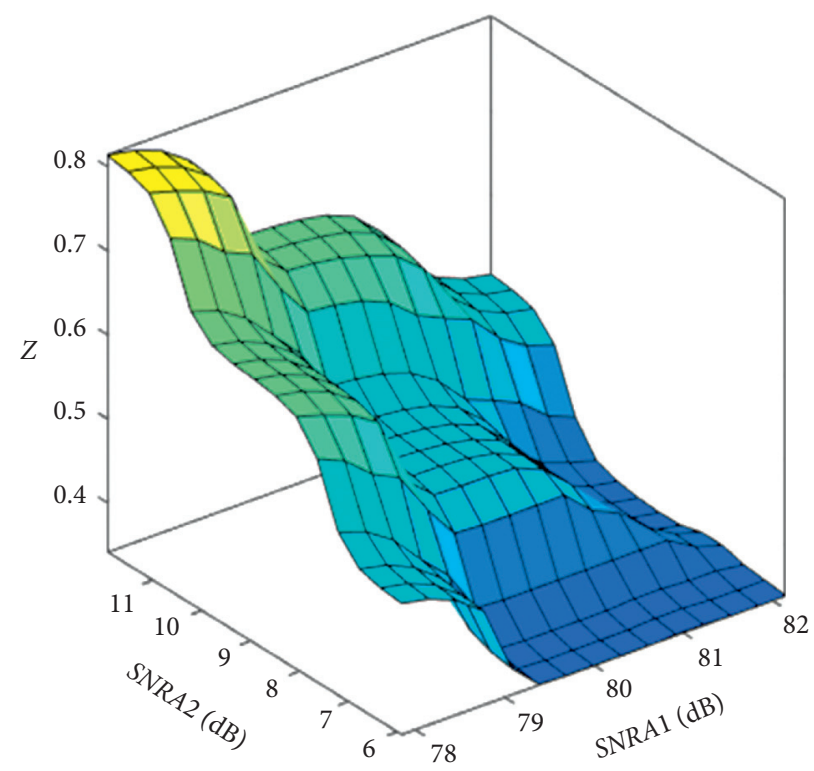

Figure 6: Plot of the $S / N$ of SNRA1 and SNRA2 vs output in the FIS system.

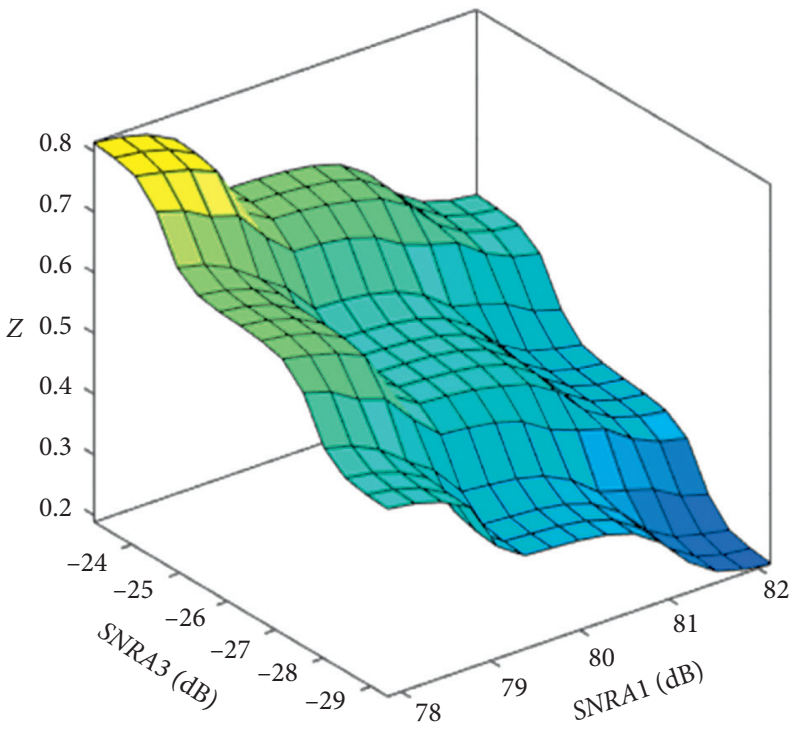

FIgURE 7: Plot of SNRA1 and SNRA3 vs output in the FIS system.

for an integration into compliant positioners in the nanoindentation tester system. (ii) An effective integration method of the Taguchi method, fuzzy logic, the response surface method, and the MFO algorithm is developed to simultaneously optimize so as to enhance the three performances for the flexure hinge. (iii) The Optimal elliptical flexure hinge has better behaviors than the initial design and other flexure hinges. (iv) The hybrid optimization approach outweighs the ASO algorithm and the GA.

Furthermore, the advantages of the present study are summarized as follows: (i) The effective use of the optimal strategy was meant to optimize the parameters of the elliptical hinge in order to enhance the three quality responses of the flexure hinge simultaneously. (ii) In the fuzzy modeling, the weight factors for each objective function were 


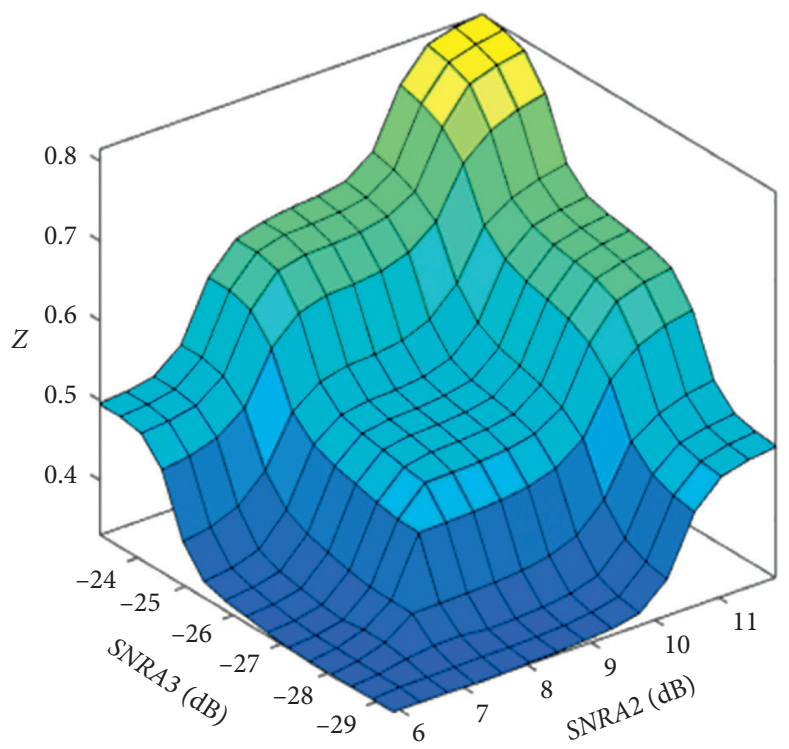

FigURE 8: Plot of SNRA2 and SNRA3 vs output in the FIS system.

SNRA1 (dB)

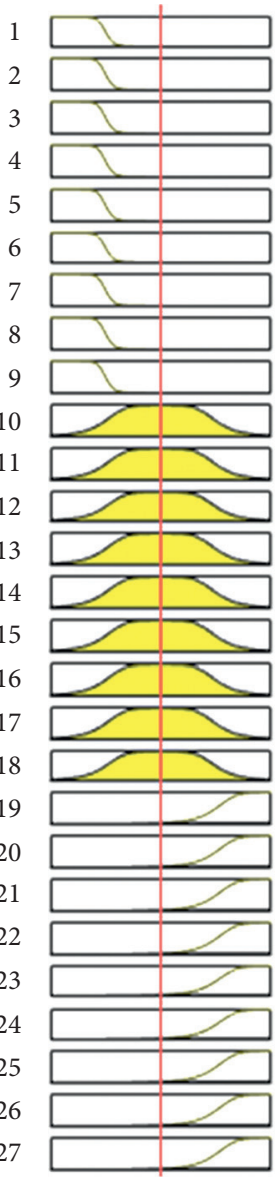

SNRA2 (dB)

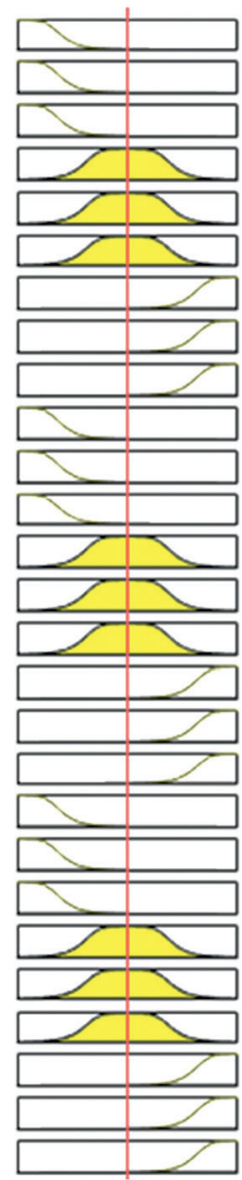

Figure 9: Twenty-seven fuzzy rules.
SNRA3 (dB)

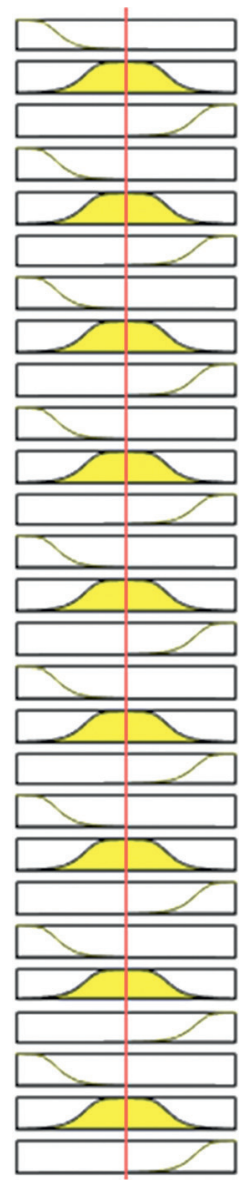

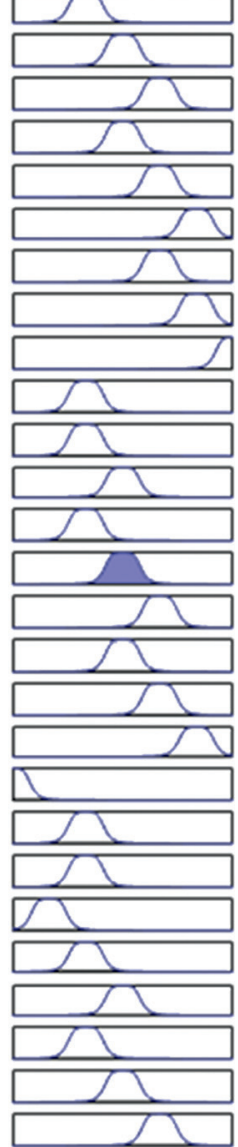


TABLE 8: ANOVA analysis.

\begin{tabular}{|c|c|c|c|c|c|c|}
\hline Source & DF & Seq SS & Contribution (\%) & Adj SS & Adj MS & $P$ value \\
\hline Model & 8 & 0.522239 & 100.00 & 0.522239 & 0.065280 & Meaningful \\
\hline Linear & 3 & 0.512998 & 98.23 & 0.218474 & 0.072825 & Meaningful \\
\hline$h$ & 1 & 0.019654 & 3.76 & 0.019654 & 0.019654 & Meaningful \\
\hline$r_{x}$ & 1 & 0.445211 & 85.25 & 0.194056 & 0.194056 & Meaningful \\
\hline$r_{y}$ & 1 & 0.048133 & 9.22 & 0.031314 & 0.031314 & Meaningful \\
\hline Square & 3 & 0.003539 & 0.68 & 0.000818 & 0.000273 & Meaningful \\
\hline$h * h$ & 1 & 0.000209 & 0.04 & 0.000209 & 0.000209 & Meaningful \\
\hline$r_{x} * r_{x}$ & 1 & 0.002699 & 0.52 & 0.000523 & 0.000523 & Meaningful \\
\hline$r_{y} * r_{y}$ & 1 & 0.000631 & 0.12 & 0.000040 & 0.000040 & Meaningful \\
\hline 2-Way interaction & 2 & 0.005702 & 1.09 & 0.005702 & 0.002851 & Meaningful \\
\hline$h * r_{x}$ & 1 & 0.003744 & 0.72 & 0.000953 & 0.000953 & Meaningful \\
\hline$h * r_{y}$ & 1 & 0.001958 & 0.38 & 0.001958 & 0.001958 & Meaningful \\
\hline Error & 0 & - & - & - & - & \\
\hline Overall & 8 & 0.522239 & 100.00 & & & \\
\hline
\end{tabular}

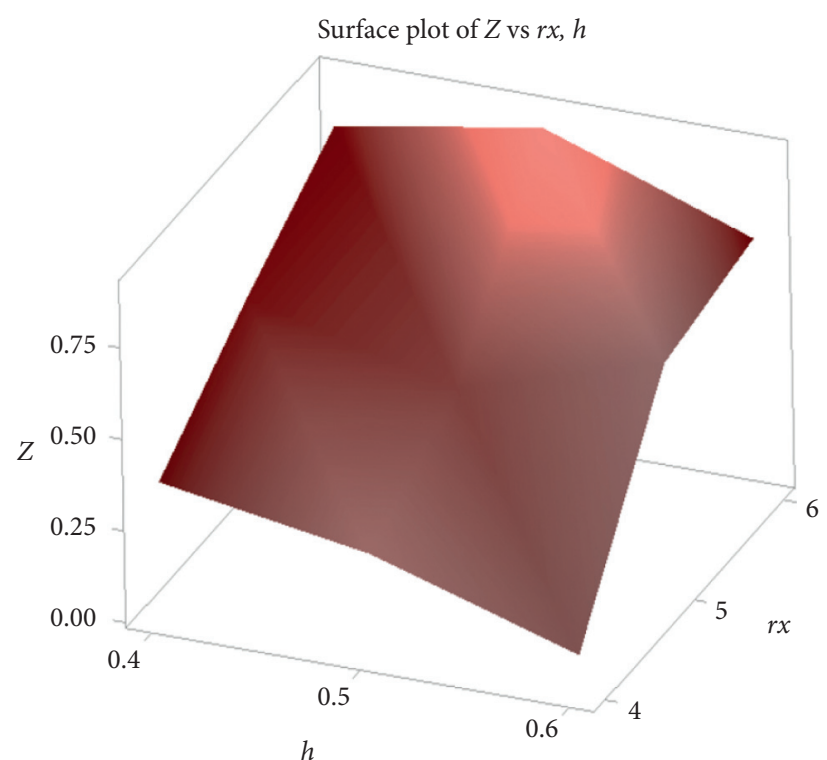

(a)

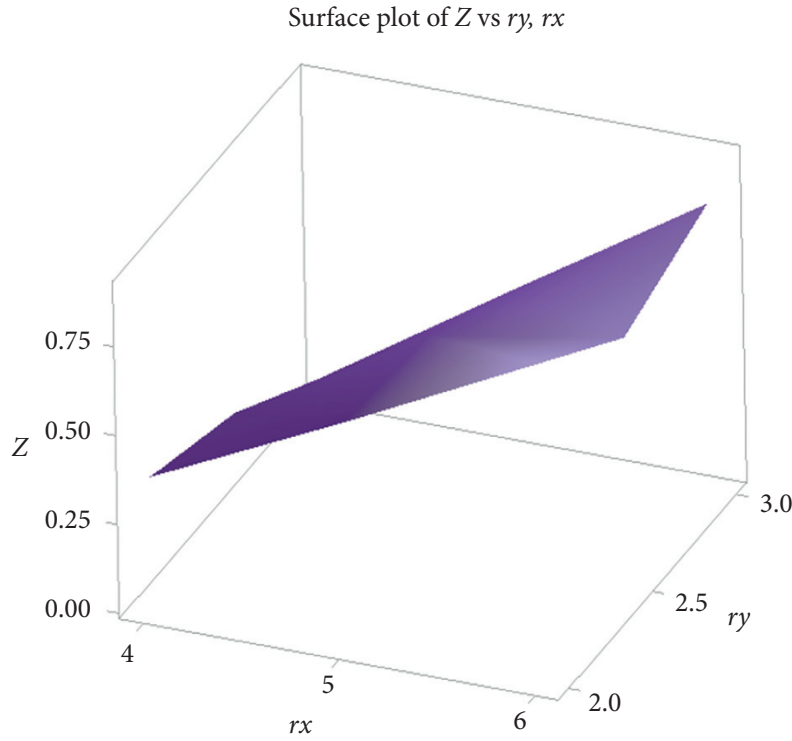

(b)

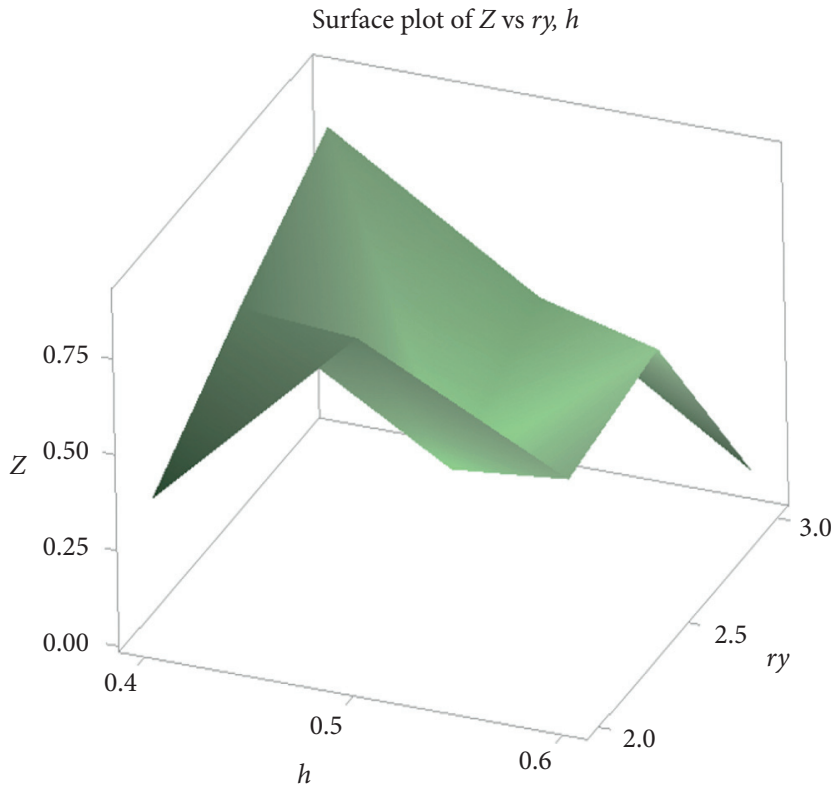

(c)

FIGURE 10: Effect diagram of the output response: (a) Influence of $h$ and $r_{x}$. (b) Influence of $r_{x}$ and $r_{y}$. (c) Influence of $h$ and $r_{y}$. 


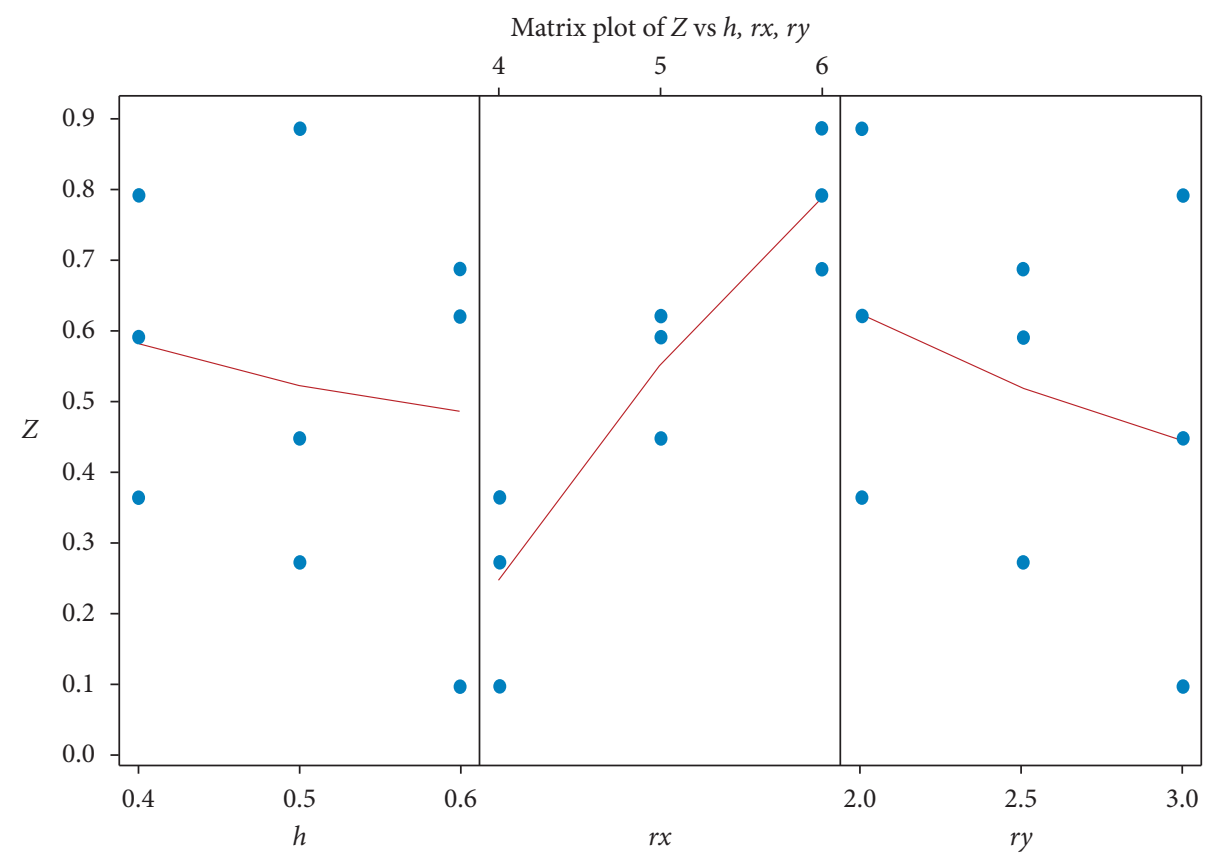

FIGURE 11: Impressibility scheme of the factors on the integrated response.

TABle 9: Used primary parameters for the MFO.

Parameters

Number of searching agents

Value

TABLE 10: Error among the predicted results and validations.

\begin{tabular}{lccc}
\hline Responses & Forecast & Verification & Error (\%) \\
\hline$y_{1}(\mathrm{~mm})$ & 0.00010944 & 0.00011715 & 6.58 \\
$y_{2}$ & 2.99349 & 2.95 & 1.47 \\
$y_{2}(\mathrm{rad})$ & 0.0520058 & 0.052 & 0.01 \\
\hline
\end{tabular}

TABLe 11: Differences between the initial design and the optimized design.

\begin{tabular}{lccc}
\hline Performance & Optimized design & Initial design & Improvement $(\%)$ \\
\hline$y_{1}(\mathrm{~mm})$ & 0.00010944 & $9.03 E-05$ & -21.19 \\
$y_{2}$ & 2.99349 & 2.71 & 10.46 \\
$y_{3}(\mathrm{rad})$ & 0.0520058 & 0.047 & 10.65 \\
\hline
\end{tabular}

TABLE 12: Comparison of the optimized hinge with the other hinges.

\begin{tabular}{|c|c|c|c|c|c|}
\hline Flexure hinges & Geometrical parameters $(\mathrm{mm})$ & $\begin{array}{l}\text { Input deflection } \\
\text { angle (degree) }\end{array}$ & $\begin{array}{l}\text { Rotation axis shift } \\
\qquad(v)(\mathrm{mm})\end{array}$ & $\begin{array}{c}\text { Safety } \\
\text { factor }(S F)\end{array}$ & $\begin{array}{l}\text { Maximal angular deflection } \\
(\varphi)(\mathrm{rad})\end{array}$ \\
\hline $\begin{array}{l}\text { Right circular } \\
\text { hinge }\end{array}$ & $\begin{aligned} H=10, h & =0.5, L_{1}=10, L_{2}=10, \\
l & =9.987, w=5\end{aligned}$ & Analysis angle $\left(1^{\circ}\right)$ & $7.2113 e-05$ & 2.11 & $x^{2}$ \\
\hline $\begin{array}{l}\text { Corner filleted } \\
\text { hinge }\end{array}$ & $\begin{array}{c}H=10, h=0.5, L_{1}=10, L_{2}=10, \\
l=10, w=5\end{array}$ & Analysis angle $\left(1^{\circ}\right)$ & 0.00022379 & 0.1 & 0.176 \\
\hline $\begin{array}{l}\text { Power function } 1 \\
(n=2)\end{array}$ & $\begin{array}{c}H=10, h=0.5, L_{1}=10, L_{2}=10, \\
l=10, w=5\end{array}$ & Analysis angle $\left(1^{\circ}\right)$ & $5.486 \mathrm{e}-05$ & 1.55 & 0.027 \\
\hline $\begin{array}{l}\text { The optimized } \\
\text { hinge }\end{array}$ & $\begin{array}{c}H=10, h=0.5, L_{l}=10, L_{2}=10 \\
l=10, w=5, r_{x}=5, r_{y}=2.5\end{array}$ & Analysis angle $\left(1^{\circ}\right)$ & 0.00010944 & 2.99349 & 0.0520058 \\
\hline
\end{tabular}


TABLE 13: Wilcoxon's comparison of the present method vs ASO.

\begin{tabular}{lccc}
\hline Number of tests & Approximated average & $P$ value & Wilcoxon statistic \\
\hline 30 & 0.0137299 & $<0.001$ & 465 \\
\hline
\end{tabular}

TABle 14: Friedman test for the integrated objective function.

\begin{tabular}{lcc}
\hline Output-integrated objective & No. & Average \\
\hline The present method & 30 & 2.76140 \\
ASO & 30 & 2.74791 \\
Total & 60 & 2.75466 \\
DF & Chi-square & $P$ value \\
1 & 30 & $<0.001$ \\
Null hypothesis & & $H_{0}:$ All treatment influences are zero \\
Substitute hypothesis & & $H_{1}:$ Not all treatment influences are zero \\
\hline
\end{tabular}

TABLE 15: Average convergence time's comparison of proposed algorithm vs the ASO and the GA.

\begin{tabular}{lc}
\hline Output-integrated objective & Average convergence time (s) \\
\hline The present method & 11.446 \\
ASO & 11.867 \\
GA & 13.159 \\
\hline
\end{tabular}

assigned automatically for the integrated equation. (iii) Mechanical researchers can assign their professional experiences for establishing a relationship between the fuzzy inputs and the fuzzy output in order to enhance the accuracy of the optimal results in the fuzzy modeling phase.

\section{Conclusions}

This article proposed an effective integration optimization method for the elliptical flexure hinge. This flexure hinge was desired to integrate into a positioner, which locates the material specimen during the nanoindentation testing. Based on the detasFLEX software, four kinds of flexure hinges, namely, right circular hinge, corner-filleted hinge, elliptical hinge, and power function hinge 1, were compared in terms of rotation axis shift, safety factor, and maximal angular deflection with the same value of the initial analysis angle. Based on the analysis results, the elliptical hinge was selected because of its beneficial features.

To simultaneously improve the small rotation axis shift, the high safety factor, and the angular deflection, the key factors of the elliptical flexure hinge were optimized by a combination of the Taguchi method, fuzzy logic reasoning, the response surface method, and the MFO algorithm. Firstly, numerical experiments are collected by the Taguchi-based detasFlex software. Secondly, three objective functions are conveyed into signal to noise so as to eradicate the unit dissimilarities. Thirdly, fuzzy modeling is proposed to interpolate three objective functions into one integrated objective function. An integrated regression equation is established utilizing the response surface method. Finally, the flexure hinge is optimized by the Moth-flame optimization algorithm. The consequences demonstrated that the optimized factors were found at $h=0.6 \mathrm{~mm}, r_{x}=6 \mathrm{~mm}$, and $r_{y}=3 \mathrm{~mm}$. Besides, the results indicated that the small rotation axis shift is $10.944 * 10^{-5} \mathrm{~mm}$, the high safety factor is 2.993 , and the angular deflection is $52.0058 * 10^{-3} \mathrm{rad}$. In addition, the sensitivity analysis and the analysis of variance were accomplished to identify meaningful influences of the input variables on the integration response.

Relying on the Wilcoxon and Friedman results, the integrated method overcomes the ASO algorithm. Furthermore, the average convergence time for 30 runs of the algorithms, namely, the integrated approach, the ASO algorithm, and the GA was compared. The result illustrated that the convergence speed of the proposed method is better than the convergence speed of the ASO algorithm and the GA. Therefore, based on the abovementioned results, the proposed integration approach is beneficial and effective to conduct the multi-objectives optimization problem for complicated engineering.

The limitations of the current study are that establishing fuzzy rules for mapping fuzzy inputs and fuzzy outputs are relatively complicated. Moreover, formation requires professional experiences and takes lots of time. To simplify the calculation for complex optimization problems, future researchers can use the adaptive network-based fuzzy inference system or other approaches to conduct optimal process. However, each approach has advantages and disadvantages for optimal calculations. Based on the real problem, the researchers should choose a suitable approach.

In the future research, an elliptical hinge will be fabricated in order to compare the optimal results. In addition, the elliptical hinge will be integrated into compliant positioners for locating material samples in the nanoindentation tester. The optimization method can be extended to solve multiple degrees of freedom positioners. Moreover, the proposed hybrid approach will be applied for solving complex multi-objective optimal problems.

\section{Data Availability}

The data used to support the findings of this study are included within the article. 


\section{Conflicts of Interest}

The authors declare that there are no conflicts of interest regarding the publication of this article.

\section{Acknowledgments}

This work was supported by the project under grant no: T2020-60TĐ funded by Ho Chi Minh City University of Technology and Education, Vietnam.

\section{References}

[1] J. J. Uicker, G. R. Pennock, J. E. Shigley, and J. M. McCarthy, "Theory of machines and mechanisms," Journal of Mechanical Design, vol. 125, 2003.

[2] Z. Hu, K. J. Lynne, S. P. Markondapatnaikuni, and F. Delfanian, "Material elastic-plastic property characterization by nanoindentation testing coupled with computer modeling," Materials Science and Engineering: A, vol. 587, pp. 268-282, 2013.

[3] W. O'Brien, "Long-range motion with nanometer precision," Photonics Spectra, Laurin Publishing Company, Pittsfield, MA, USA, 2005.

[4] J. Nohava, N. X. Randall, and N. Conté, "Novel ultra nanoindentation method with extremely low thermal drift: principle and experimental results," Journal of Materials Research volume, vol. 24, 2009.

[5] H. Huang, C. Shi, H. Zhao, and L. Zhang, "Influence of friction on the residual morphology, the penetration load and the residual stress distribution of a Zr-based bulk metallic glass," AIP Advances, vol. 3, 2013.

[6] A. S. Putra, S. Huang, K. K. Tan, S. K. Panda, and T. H. Lee, "Design, modeling, and control of piezoelectric actuators for intracytoplasmic sperm injection," IEEE Transactions on Control Systems Technology, vol. 15, no. 5, pp. 879-890, 2007.

[7] Q. Xu, "Design and testing of a novel multi-stroke micropositioning system with variable resolutions," Review of Scientific Instruments, vol. 85, p. 25002, 2014.

[8] H. Kim, J. Kim, D. Ahn, and D. Gweon, "Development of a nanoprecision 3-DOF vertical positioning system with a flexure hinge," IEEE Transactions on Nanotechnology, vol. 12, no. 2, pp. 234-245, 2013.

[9] B. Ding, Z. Yang, and Y. Li, "Design of flexure-based modular architecture micro-positioning stage," Microsystem Technologies, vol. 26, no. 9, pp. 2893-2901, 2020.

[10] M. Ling, L. L. Howell, J. Cao, and G. Chen, "Kinetostatic and dynamic modeling of flexure-based compliant mechanisms: a survey," Applied Mechanics Reviews, vol. 72, 2020.

[11] B. Ding, Z.-X. Yang, X. Xiao, and G. Zhang, "Design of reconfigurable planar micro-positioning stages based on function modules," IEEE Access, vol. 7, pp. 15102-15112, 2019.

[12] S. P. Wadikhaye, Y. K. Yong, and S. O. R. Moheimani, "Design of a compact serial-kinematic scanner for high-speed atomic force microscopy: an analytical approach," Micro \& Nano Letters, vol. 7, no. 4, pp. 309-313, 2012.

[13] M. Gauthier and E. Piat, "Control of a particular micro-macro positioning system Applied to cell micromanipulation," IEEE Transactions on Automation Science and Engineering, vol. 3, no. 3, pp. 264-271, 2006.

[14] W.-L. Zhu, Z. Zhu, P. Guo, and B.-F. Ju, "A novel hybrid actuation mechanism based XY nanopositioning stage with totally decoupled kinematics," Mechanical Systems and Signal Processing, vol. 99, pp. 747-759, 2018.
[15] K. Lu, J. Zhang, W. Chen, J. Jiang, and W. Chen, “A monolithic microgripper with high efficiency and high accuracy for optical fiber assembly," in Proceedings of the 2014 9th IEEE Conference on Industrial Electronics and Applications, ICIEA 2014, pp. 1942-1947, Hangzhou, China, June 2014.

[16] H. Huang, H. Zhao, J. Mi et al., "A novel and compact nanoindentation device for in situ nanoindentation tests inside the scanning electron microscope," AIP Advances, vol. 2, 2012.

[17] H. Huang, H. Zhao, J. Mi et al., "Experimental research on a modular miniaturization nanoindentation device," Review of Scientific Instruments, vol. 82, 2011.

[18] N. Lobontiu and E. Garcia, "Analytical model of displacement amplification and stiffness optimization for a class of flexurebased compliant mechanisms," Computers \& Structures, vol. 81, no. 32, pp. 2797-2810, 2003.

[19] Y.-1. Yang, Y.-d. Wei, J.-q. Lou, G. Tian, X.-w. Zhao, and L. Fu, "A new piezo-driven microgripper based on the doublerocker mechanism," Smart Materials and Structures, vol. 24, no. 7, p. 075031, 2015.

[20] Q. Xu and Y. Li, "Analytical modeling, optimization and testing of a compound bridge-type compliant displacement amplifier," Mechanism and Machine Theory, vol. 46, no. 2, pp. 183-200, 2011.

[21] P. Liu and P. Yan, "A new model analysis approach for bridgetype amplifiers supporting nano-stage design," Mechanism and Machine Theory, vol. 99, pp. 176-188, 2016.

[22] Y. Ni, Z. Deng, X. Wu, J. Li, and L. Li, "Quasi-static and modal analysis of bridge-type compliant mechanism with flexure hinges," in Proceedings of the 2013 Second International Conference on Robot, Vision and Signal Processing (RVSP 2013), pp. 102-106, Kitakyushu, Japan, December 2013.

[23] M. Ling, J. Cao, M. Zeng, J. Lin, and D. J. Inman, "Enhanced mathematical modeling of the displacement amplification ratio for piezoelectric compliant mechanisms," Smart Materials and Structures, vol. 25, 2016.

[24] F. Wang, C. Liang, Y. Tian, X. Zhao, and D. Zhang, "Design and control of a compliant microgripper with a large amplification ratio for high-speed micro manipulation," IEEE/ ASME Transactions on Mechatronics, vol. 21, no. 3, pp. 1262-1271, 2016.

[25] H. Tang, Y. Li, and X. Xiao, "Development and assessment of a novel hydraulic displacement amplifier for piezo-actuated large stroke precision positioning," in Proceedings of the 2013 IEEE International Conference on Robotics and Automation, pp. 1409-1414, Karlsruhe, Germany, May 2013.

[26] S. H. Chang and B. C. Du, "A precision piezodriven micropositioner mechanism with large travel range," Review of Scientific Instruments, vol. 69, no. 4, pp. 1785-1791, 1998.

[27] Q. Xu, "Structure design of a new compliant gripper based on Scott-Russell mechanism," in Proceedings of the 2013 IEEE International Conference on Robotics and Biomimetics (ROBIO 2013), Shenzhen, China, December 2013.

[28] N. Lobontiu and J. S. N. Paine, "Design of circular crosssection corner-filleted flexure hinges for three-dimensional compliant mechanisms," Journal of Mechanical Design, vol. 124, 2002.

[29] Y. K. Yong, T.-F. Lu, and D. C. Handley, "Review of circular flexure hinge design equations and derivation of empirical formulations," Precision Engineering, vol. 32, no. 2, pp. 63-70, 2008.

[30] N. Lobontiu, M. Cullin, M. Ali, and J. M. F. Brock, “A generalized analytical compliance model for transversely 
symmetric three-segment flexure hinges," Review of Scientific Instruments, vol. 82, pp. 1-10, 2011.

[31] N. Xu, M. Dai, and X. Zhou, "Analysis and design of symmetric notch flexure hinges," Advances in Mechanical Engineering, vol. 9, pp. 1-12, 2017.

[32] X. Zhang and B. Zhu, Topology Optimization of Compliant Mechanisms, Springer, Berlin, Germany, 2018.

[33] B. Zhu, X. Zhang, M. Liu, Q. Chen, and H. Li, "Topological and shape optimization of flexure hinges for designing compliant mechanisms using the level set method," Chinese Journal of Mechanical Engineering, vol. 32, 2019.

[34] S. Zelenika, M. G. Munteanu, and F. De Bona, "Optimized flexural hinge shapes for microsystems and high-precision applications," Mechanism and Machine Theory, vol. 44, no. 10, pp. 1826-1839, 2009.

[35] Z. Yang, Y. Bai, and X. Chen, "Simultaneous optimal design of topology and size for a flexure-hinge-based guiding mechanism to minimize mass under stiffness and frequency constraints," Engineering Optimization, vol. 49, no. 6, pp. 948-961, 2017.

[36] J. A. Ghani, I. A. Choudhury, and H. H. Hassan, "Application of Taguchi method in the optimization of end milling parameters," Journal of Materials Processing Technology, vol. 145, no. 1, pp. 84-92, 2004.

[37] H. Golpîra, H. Bevrani, and H. Golpîra, "Application of GA optimization for automatic generation control design in an interconnected power system," Energy Conversion and Management, vol. 52, no. 5, pp. 2247-2255, 2011.

[38] A. H. Gandomi, X.-S. Yang, and A. H. Alavi, "Cuckoo search algorithm: a metaheuristic approach to solve structural optimization problems," Engineering with Computers, vol. 29, no. 1, pp. 17-35, 2013.

[39] A. Chander, A. Chatterjee, and P. Siarry, "A new social and momentum component adaptive PSO algorithm for image segmentation," Expert Systems with Applications, vol. 38, no. 5, pp. 4998-5004, 2011.

[40] R. V. Rao and V. Patel, "Multi-objective optimization of heat exchangers using a modified teaching-learning-based optimization algorithm," Applied Mathematical Modelling, vol. 37, no. 3, pp. 1147-1162, 2013.

[41] R. V. Rao, V. J. Savsani, and D. P. Vakharia, "Teachinglearning-based optimization: a novel method for constrained mechanical design optimization problems," Computer-Aided Design, vol. 43, no. 3, pp. 303-315, 2011.

[42] R. V. Rao, V. J. Savsani, and D. P. Vakharia, "TeachingLearning-Based Optimization: an optimization method for continuous non-linear large scale problems," Information Science, vol. 183, 2012.

[43] W. Zhao, L. Wang, and Z. Zhang, "Atom search optimization and its application to solve a hydrogeologic parameter estimation problem," Knowledge-Based Systems, vol. 163, pp. 283-304, 2019.

[44] R. V. Rao and R. B. Pawar, "Constrained design optimization of selected mechanical system components using Rao algorithms," Applied Soft Computing, vol. 89, p. 106141, 2020.

[45] S. Mirjalili, "Moth-flame optimization algorithm: a novel nature-inspired heuristic paradigm," Knowledge-Based Systems, vol. 89, pp. 228-249, 2015.

[46] T. P. Dao, "Multiresponse optimization of a compliant guiding mechanism using hybrid taguchi-grey based fuzzy logic approach," Mathematical Problems in Engineering, vol. 2016, Article ID 5386893, 17 pages, 2016.

[47] T.-H. Hou, C.-H. Su, and W.-L. Liu, "Parameters optimization of a nano-particle wet milling process using the Taguchi method, response surface method and genetic algorithm," Powder Technology, vol. 173, no. 3, pp. 153-162, 2007.

[48] H. Garg, "A hybrid PSO-GA algorithm for constrained optimization problems," Applied Mathematics and Computation, vol. 274, pp. 292-305, 2016.

[49] H. Garg, "A hybrid GSA-GA algorithm for constrained optimization problems," Information Sciences, vol. 478, pp. 499-523, 2019.

[50] H. Garg, "A hybrid GA-GSA algorithm for optimizing the performance of an industrial system by utilizing uncertain data," Handbook of Research on Artificial Intelligence Techniques and Algorithms, pp. 620-654, 2014.

[51] D. N. Le, G. N. Nguyen, H. Garg, Q. T. Huynh, T. N. Bao, and N. N. Tuan, "Optimizing bidders selection of multi-round procurement problem in software project management using parallel max-min ant system algorithm," Computers, Materials and Continua, vol. 66, pp. 993-1010, 2021.

[52] T.-P. Dao, S.-C. Huang, and P. T. Thang, "Hybrid Taguchicuckoo search algorithm for optimization of a compliant focus positioning platform," Applied Soft Computing, vol. 57, pp. 526-538, 2017.

[53] S. Xiao, Y. Li, and X. Zhao, "Optimal design of a novel microgripper with completely parallel movement of gripping arms," in Proceedings of the IEEE Conference on Robotics, Automation and Mechatronics, RAM, pp. 35-40, Qingdao, China, September 2011.

[54] M. P. Dang, H. G. Le, N. Le Chau, and T. P. Dao, A MultiObjective Optimization Design for a New Linear Compliant Mechanism, Springer, Berlin, Germany, 2020.

[55] N. T. Tran, N. Le Chau, and T. P. Dao, "A hybrid computational method of desirability, fuzzy logic, ANFIS, and LAPO algorithm for multiobjective optimization design of Scott russell compliant mechanism," Mathematical Problems in Engineering, vol. 2020, Article ID 3418904, 28 pages, 2020.

[56] M. Aamir, S. Tu, M. Tolouei-Rad, K. Giasin, and A. Vafadar, "Optimization and modeling of process parameters in multihole simultaneous drilling using taguchi method and fuzzy logic approach," Materials (Basel), vol. 13, 2020.

[57] S. Henning, S. Linß, and L. Zentner, "DetasFLEX-a computational design tool for the analysis of various notch flexure hinges based on non-linear modeling," Mechanical Sciences, vol. 9, no. 2, pp. 389-404, 2018.

[58] M. A. Díaz-Cortés, E. Cuevas, J. Gálvez, and O. Camarena, “A new metaheuristic optimization methodology based on fuzzy logic," Applied Soft Computing, vol. 61, pp. 549-569, 2017.

[59] L. Wang, H. Garg, and N. Li, "Pythagorean fuzzy interactive Hamacher power aggregation operators for assessment of express service quality with entropy weight," Soft Computing, vol. $25,2021$.

[60] H. Garg, Z. Ali, and T. Mahmood, "Generalized dice similarity measures for complex Q-Rung orthopair fuzzy sets and its application," Complex \& Intelligent Systems, vol. 2020, pp. $1-20,2020$

[61] G. I. Sayed and A. E. Hassanien, "A hybrid SA-MFO algorithm for function optimization and engineering design problems," Complex \& Intelligent Systems, vol. 4, no. 3, pp. 195-212, 2018.

[62] R. Barham, A. Sharieh, and A. Sleit, "Moth flame optimization based on golden section search and its application for link prediction problem," Modern Applied Science, vol. 13, 2018.

[63] M. Shehab, L. Abualigah, H. Al Hamad, H. Alabool, M. Alshinwan, and A. M. Khasawneh, "Moth-flame optimization algorithm: variants and applications," Neural Computing and Applications, vol. 32, no. 14, pp. 9859-9884, 2020. 